\title{
Shock séptico en pediatría II. Enfoque actual en el diagnóstico y tratamiento
}

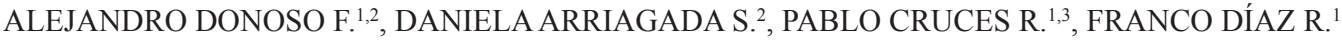 \\ 1. Área de Cuidados Críticos. Unidad de Gestión Clínica de Niño. Hospital Padre Hurtado. \\ 2. Programa de Medicina Intensiva en Pediatría. Facultad de Medicina Clínica Alemana. Universidad del Desarrollo. \\ 3. Centro de Investigación de Medicina Veterinaria, Escuela de Medicina Veterinaria, Facultad de Ecología y \\ Recursos Naturales, Universidad Andrés Bello, Santiago, Chile.
}

\begin{abstract}
Septic shock in pediatrics II. Current concepts in diagnosis and treatment

Although the basic concepts of diagnosis and therapy of the child with septic shock have remained similar over time, it is undeniable that in recent decades, new and important concepts have been added, and any treating physician either at the Emergency Department or Intensive Care Unit should be fully aware of them. This second part discusses the similarities and differences between pediatric and adult populations, the utility of metabolic resuscitation goals, as well as the initial therapeutic approach in septic patients. The most important concepts of this work make reference to the differences between children and adults with septic shock, specifically regarding to pathophysiology, clinical presentation and treatment. Volume expansion and vasoactive drugs are crucial if there is no response to fluid resuscitation. The therapeutic management should focus on finding the normalization of macrohemodynamic and systemic perfusion targets. Common observation mistakes in the initial treatment of septic patients should be avoided. This condition has a high incidence and mortality rate; therefore an early and aggressive treatment is essential.
\end{abstract}

(Key words: Sepsis, septic shock, resuscitation, capillary refill time, pediatric emergency).

Rev Chil Pediatr 2013; 84 (6): 606-615

\section{RESUMEN}

Aunque los principios básicos para el diagnósticoy los pilares terapéuticos del niño con shock séptico se mantienen en el tiempo, es innegable que en las últimas décadas se han incorporado nuevos y trascendentes conceptos, siendo importante que el médico tratante en el Servicio de Urgencia tenga conocimiento a cabalidad de ellos. En la segunda parte de esta actualización se discuten las similitudes y diferencias entre la población infantil y adulta, la utilidad de las metas de reanimación metabólicas, como también el enfoque terapéutico inicial en el paciente séptico. Los conceptos más importantes revisados se refieren a las diferencias entre el niño y adulto con shock séptico, especialmente en la fisiopatología, clínica y tratamiento. Se recalca la importancia de expansión de la volemia y el uso de drogas vasoactivas si no hay respuesta a fluidos de reanimación. El manejo terapéutico debe estar

Recibido el 13 de diciembre de 2012, devuelto para corregir el 16 de julio de 2013, segunda versión el 21 de septiembre de 2013, aceptado para publicación el 21 de octubre de 2013.

Este trabajo cumple con los requisitos sobre consentimiento /asentimiento informado, comité de ética, financiamiento, estudios animales y sobre la ausencia de conflictos de intereses según corresponda.

Correspondencia a:

Dr. Alejandro Donoso F.

E-mail: adonoso@hurtadohosp.cl 
orientado a la búsqueda de la normalización de metas macrohemodinámicas y de perfusión sistémica. Se deben evitar los errores más frecuentes de observar en el tratamiento inicial del paciente séptico. Esta entidad presenta una elevada incidencia y mortalidad, por lo cual el manejo precoz y agresivo es de máxima importancia en pediatría.

(Palabras clave: sepsis, shock séptico, reanimación, tiempo de llene capilar, urgencia pediátrica).

Rev Chil Pediatr 2013; 84 (6): 606-615

\section{Introducción}

La sepsis grave (SG) y el shock séptico (SS) son importantes problemas de salud pública, constituyéndose en entidades catastróficas con una importante morbimortalidad asociada ${ }^{1}$.

A pesar de la existencia de similitudes en el enfoque del paciente adulto y pediátrico séptico, existen diferencias claves en relación a la fisiopatología, formas de presentación clínica y opciones terapéuticas a emplear ${ }^{2}$.

$\mathrm{Su}$ diagnóstico precoz, tratamiento agresivo en forma protocolizada y orientado por metas son esenciales para lograr un resultado satisfactorio. Es trascendental la evaluación del paciente mediante el examen físico en la búsqueda de la respuesta, o falta de ésta, a las decisiones terapéuticas efectuadas.

El objetivo de la segunda parte de esta publicación es destacar las características propias del SS en este grupo etario y actualizar el estado del tratamiento inicial al que debe ser sometido el paciente, particularmente cuando los pediatras deben intervenir en el ámbito preunidad de cuidados intensivos (UCI).

\section{Similitudes del shock séptico en población infantil y adulta}

El pronóstico de los pacientes para ambos grupos etarios depende del tiempo transcurrido, destacando así la importancia del reconocimiento y tratamiento precoz del SS, lo cual ha sido demostrado mediante evidencia contundente. En la población infantil, por cada hora transcurrida sin usar las guías Pediatric Advanced Life Support (PALS)/Advanced Pediatric Life Support (APLS) la mortalidad aumenta en un $40 \%{ }^{3}$. De la misma manera, la presencia de hipotensión arterial incrementa la mortalidad a un $4,4 \%$, el retardo del tiempo de llene capilar a un 7,6\%, y si ambas con- diciones están presentes aumenta a un 27\%. Reforzando el mismo concepto, Kumar et $\mathrm{al}^{4}$, identificaron una disminución en la sobrevida en un $7,6 \%$ por cada hora transcurrida de retardo sin recibir antibioterapia. Asimismo, Barie et $\mathrm{al}^{5}$, evaluaron un grupo de pacientes adultos sometidos a cirugía, lográndose identificar que aquellos pacientes en los cuales se había efectuado remoción del foco infeccioso, la sobrevida aumentaba a un 95\%, en comparación con aquellos en quienes no se realizó, cuya sobrevida fue sólo de un $4 \%$.

Por lo tanto, el tiempo de diagnóstico e inicio de tratamiento (resucitación, uso precoz de antibiótico y remoción del nido infeccioso), son las piedras angulares en el manejo del SS.

Otra similitud entre la población adulta e infantil es que luego de la resucitación inicial, se observa el desarrollo de un estado de inmunosupresión (Compensatory Anti-inflammatory Response Syndrome, CARS). Este es un fenómeno adaptativo que "apaga" la respuesta inflamatoria, pero puede contribuir a una mayor susceptibilidad a infecciones por condicionar un estado de inmunoparálisis, la cual juega un rol clave en el síndrome de disfunción orgánica múltiple (SDOM). En los hallazgos necrópsicos de pacientes con SDOM destaca la depleción linfoide ocasionada por apoptosis, lo que junto a la falla de la terapia empleada en múltiples ensayos con agentes anti-inflamatorios, ha llevado a cuestionar si la mortalidad en esta condición es secundaria a una respuesta anti-inflamatoria no controlada más que a un estado pro-inflamatorio ${ }^{2}$.

\section{Diferencias del shock séptico en población infantil y adulta}

La respuesta cardiovascular a la SG es compleja y variable. En el paciente adulto el patrón hemodinámico característico es el de 
un estado hiperdinámico asociado a diversos grados de parálisis vasomotora.

La disfunción miocárdica en ocasiones presente en adultos, es compensada mediante dos mecanismos, como son la taquicardia sinusal y dilatación ventricular, presentando un peor pronóstico vital aquellos pacientes que no logran desarrollar en forma eficiente estos mecanismos.

En contraste, el SS en el paciente pediátrico se asocia muy frecuentemente a hipovolemia grave, la que ocasiona una caída del volumen eyectivo y el que habitualmente responde a una adecuada resucitación con fluidos. Sin embargo, si persiste el shock, su respuesta hemodinámica es distinta a la descrita en adultos caracterizándose por un bajo gasto cardíaco (GC), hipodébito y elevada resistencia vascular, de manera que es la disponibilidad de oxígeno (oxygen delivery, $\mathrm{DO}_{2}$ ) y no su extracción (oxygen consumption, $\left[\mathrm{VO}_{2}\right] / \mathrm{DO}_{2}$ ), el principal determinante del consumo de oxígeno. Aproximadamente el $50 \%$ de los niños presentan este patrón hemodinámico el cual es conocido como SS frío ${ }^{6-8}$.

Otra característica a tener en consideración es la limitada reserva cardíaca de los niños, por ende, la taquicardia no es un mecanismo compensatorio efectivo (compromiso del tiempo de llenado diastólico) y así entonces, la respuesta ante una caída del GC es la vasoconstricción periférica, la que al perdurar en el tiempo puede ser finalmente perjudicial. Esto explica porque la hipotensión arterial es un signo tardío en el shock séptico en la población pediátrica.

Cabe destacar lo que ocurre en el período neonatal, ya que existe una transición fisiológica desde la etapa fetal a la neonatal. La respuesta hemodinámica ha sido menos caracterizada en neonatos (prematuros o de término) y sus anormalidades hemodinámicas son mucho más variables ${ }^{8}$. Factores que dan cuenta de estas diferencias incluyen la estructura y funcionalidad de los cardiomiocitos y su limitada capacidad para aumentar el volumen eyectivo y su contractilidad ${ }^{9}$. En relación a la fisiología del neonato, se debe recordar que un $85 \%$ de la circulación fetal sufre un by pass a nivel pulmonar debido a su paso por el foramen oval y ductus arterioso, que se mantienen por la presión suprasistémica de la arteria pulmonar (AP). En neonatos sépticos, tanto la hipoxia como la acidosis aumentan la resistencia y presión de la AP (aumento post carga VD), ocasionando una persistencia de la circulación fetal, lo que finalmente termina en falla cardía$\mathrm{ca}^{2}$. Es en este contexto donde juegan un rol las terapias destinadas a disminuir la presión de la AP como lo son el óxido nítrico inhalado (NOi) y los inhibidores de la fosfodiesterasa.

Finalmente, la persistencia del ductus arterioso (DAP) e hipertensión pulmonar neonatal son condiciones clínicas que pueden modificar el manejo de la hipotensión arterial e hipoxemia. Así, en pacientes prematuros con DAP, una administración agresiva de fluidos puede ocasionar sobrecarga de líquidos, edema pulmonar o falla cardíaca. Por el contrario, en niños de término con hipertensión pulmonar grave el empleo de fluidos y fármacos vasoactivos para mantener una presión arterial normal puede ser beneficiosa por la reducción del shunt de derecha a izquierda y mejoría en la oxigenación.

\section{Utilidad de la medición de lactato en el servicio de urgencia}

Clásicamente, la hiperlactacidemia es considerada como "un testigo" del metabolismo anaerobio, representando el desbalance entre $\mathrm{DO}_{2}$ y $\mathrm{VO}_{2}$, considerándose anormal un valor por sobre $>4 \mathrm{mmol} / \mathrm{L}^{10}$.

Numerosos estudios han establecido el uso del lactato como marcador diagnóstico, terapéutico y pronóstico ${ }^{11-16}$. Los niveles de lactato al ingreso están fuertemente asociados a pronóstico ${ }^{12}$, empero, la medición de niveles seriados tiene mayor valor que una medición única aislada ${ }^{17}$.

En la población pediátrica, son escasos los trabajos publicados y a la vez con resultados contradictorios. En forma independiente, Duke et al y Koliski et al ${ }^{18,19}$, reportaron que el lactato es buen predictor de mortalidad, mientras que Hatherill et $\mathrm{al}^{20}$, no encontraron diferencias significativas en los niveles de lactato entre los sobrevivientes y no sobrevivientes. Recientemente en un estudio prospectivo realizado en un servicio de urgencia en pacientes pediátricos con síndrome de respuesta infla- 
matoria sistémica (SRIS) ${ }^{21}$ se encontró que un $7,5 \%$ de los pacientes desarrollaban hiperlactacidemia, describiéndose para este grupo un mayor riesgo de disfunción orgánica (RR 5,5\% [IC 95\% 1,9-16]). Los autores concluyen que la medición de los niveles de lactato sanguíneo en conjunto con la evaluación clínica puede mejorar la identificación precoz de los pacientes con sepsis que requieren resucitación.

Finalmente, en una cohorte de 30 pacientes pediátricos con SS se demostró que los pacientes que fallecieron tenían mayores niveles de lactato (> $>5 \mathrm{~mol} / \mathrm{L}$ ) al ingreso, a las 12 y $24 \mathrm{~h}$ que los sobrevivientes ${ }^{22}$.

\section{Saturación $\mathrm{O}_{2}$ en vena cava superior $\left(\mathrm{SvcO}_{2}\right)$}

Se ha constituido en una meta emergente de reanimación. En palabras sencillas, la saturación venosa mixta de oxígeno $\left(\mathrm{SvO}_{2}\right)$ muestra el valor de oxigeno residual que retorna al corazón, luego de la extracción tisular ${ }^{23}$. Su valor está determinado por el equilibrio entre $\mathrm{DO}_{2}$ $\mathrm{y} \mathrm{VO}_{2}$.

La cateterización venosa central (acceso supra-diafragmático) y la medición de la saturación venosa central $\left(\mathrm{SvcO}_{2}\right)$ es habitualmente parte de la monitorización estándar del paciente críticamente enfermo. Una $\mathrm{SvcO}_{2}$ de $70 \%$ se considera como la meta mínima en esta localización. Se ha podido constatar una buena correlación entre $\mathrm{SvcO}_{2}$ y $\mathrm{SvO}_{2}{ }^{24}$.

Una interesante comunicación evaluó el efecto de la intubación endotraqueal en la $\mathrm{SvcO}_{2}$, señalando que ésta aumenta significativamente tanto en pacientes sépticos y no sépticos. En casi un tercio de los pacientes esta exclusiva maniobra incrementó la $\mathrm{SvcO}_{2}$ a niveles considerados como una meta de resucitación $(>70 \%)^{25}$.

\section{Enfoque inicial del paciente séptico}

\section{"Tiempo es tejido"}

El shock es una causa importante y a su vez prevenible, de morbimortalidad en niños que ingresan a los servicios de urgencia ${ }^{26}$, por lo que el rol del equipo de salud a este nivel es trascendental para lograr una mejor sobrevida $\mathrm{y}$ pronóstico funcional. Este equipo debe estar entrenado para reconocer, priorizar (triage) como también tratar en forma precoz y adecuada al paciente en shock en su primera hora o hasta que se traslade a $\mathrm{UCI}^{27}$. El empleo de guías para protocolizar el uso de fluidos, antibióticos e inótropos durante la primera hora de atención puede reducir la mortalidad y secuelas neurológicas ${ }^{26}$. Cruz et $\mathrm{al}^{28}$, recientemente comunicaron la implementación de un protocolo de tratamiento guiado por metas de reanimación, resultando en una disminución en el tiempo de administración de fluidos y antibióticos, lo cual mejoró la sobrevida de los pacientes.

Buscando este mismo objetivo, Larsen et $\mathrm{al}^{29}$, evaluaron la instauración de un protocolo para el reconocimiento temprano del SS y la utilización de guías de tratamiento en un servicio de urgencia. El uso de éstas fue asociada con un aumento en el registro de los signos vitales, disminución en el tiempo de hospitalización y de la mortalidad en aquellos pacientes que recibieron los tres elementos considerados claves en el tratamiento (reanimación con fluidos en la primera hora, medición de niveles de lactato y administración de antibióticos dentro de las tres primeras horas).

El reconocimiento de la sepsis en pacientes pediátricos puede ser difícil en ocasiones, ya que habitualmente la tríada inflamatoria de fiebre, taquicardia y vasodilatación es común de observar en pacientes con infecciones benignas. Así, los signos clínicos de shock pueden aparecer y no necesariamente estar presente al momento de la consulta. Recientemente Fisher et $\mathrm{al}^{30}$, en 147 niños con shock (57\% séptico), encontraron que un $15 \%$ de los pacientes se presentaron sin signos clínicos de shock y evolucionaron con deterioro clínico durante la permanencia en la unidad de emergencia. Es importante destacar que en los pacientes que empeoraron, la taquicardia estaba presente sólo en un tercio de ellos. Esto enfatiza la importancia de la constante evaluación de los pacientes con SS dado el carácter evolutivo de esta condición.

El SS debe ser sospechado cuando los niños con esta triada clínica presentan, además, alteración del estado mental y/o signos de compromiso de la perfusión tisular ${ }^{26}$. En neonatos es aún más difícil de reconocer ya que los sín- 
tomas y signos de SS pueden ser simuladas por otras condiciones tales como shock cardiogénico o debut de errores innatos del metabolismo. Como hemos señalado previamente, la hipotensión arterial es un signo tardío, por lo que su presencia no es necesaria para el diagnóstico de SS. Son estas manifestaciones clínicas inespecíficas las que nos obligan a mantener siempre un elevado índice de sospecha.

Finalmente, los signos clínicos de shock observados van a depender del patrón hemodinámico predominante en ese momento en el paciente (tabla 1). No obstante, es conocido que la habilidad del clínico para juzgar los parámetros hemodinámicos es pobre ${ }^{31}$.

\section{Reanimación precoz (primera hora)}

Como ya se mencionó, el pronóstico depende no sólo de la precocidad del diagnóstico sino también de la premura con que se inicia el tratamiento ${ }^{32}$. La reanimación guiada por metas durante la primera hora está orientada a la corrección de la perfusión tisular y por ende de la hipoxia global, lo que permite un restablecimiento de la función celular y la homeostasis metabólica ${ }^{27,33,34}$. Las metas previamente señaladas deben adecuarse a la realidad local (tabla 2).

Han et $\mathrm{al}^{35}$, reportaron un aumento de la mortalidad por cada hora que el paciente permanecía hipotenso o con un tiempo de llenado capilar $>2$ ". Por su parte, Booy et al ${ }^{36}$, demostraron que con educación comunitaria, en relación al reconocimiento y tratamiento precoz de la enfermedad meningocócica, como también mediante la creación de un equipo de transporte especializado, se logró una reducción de la mortalidad de $22 \%$ a $2 \%$. Otro pilar fundamental en el tratamiento inicial es la remoción del foco infeccioso y el inicio precoz de antibióticos. Estas medidas han demostrado mejorar la sobrevida de los pacientes sépticos.

La relevancia de la reanimación guiada por metas quedó demostrada en el clásico trabajo de Rivers et $\mathrm{al}^{24}$, en el que comunicaron una reducción de la mortalidad en pacientes adultos sépticos $(46,5 \%$ a $30,5 \%)$ cuando se utilizaba la normalización de la presión arterial y saturación venosa central $\geq 70 \%$ como meta tera- péutica luego de 6 h de reanimación. Recientemente Oliveira et $\mathrm{al}^{37}$, efectuaron un trabajo similar para la población pediátrica, donde se evaluó el rol de la reanimación guiada por metas dirigido a mantener una saturación venosa central $\geq 70 \%$ por $72 \mathrm{~h}$. Hubo una reducción de la mortalidad de $40 \%$ a $12 \%$ a los 28 días.

\section{Importancia del tiempo de llenado capilar}

El tiempo que se demora en recuperar el color rosado la piel luego de liberar la compresión externa del lecho capilar, se llama tiempo de llenado capilar (TLLC) $)^{38}$. El TLLC es dependiente de la edad y en niños se considera normal hasta 2 segundos $^{39}$, mientras que en neonatos hasta 3 segundos es el límite superior normal $^{40}$. Sin embargo, en un reciente estudio en donde se examinaron 92 niños sanos entre 0 y 12 años, se encontró a una minoría de ellos con un TLLC de 3 segundos ${ }^{41}$.

Los determinantes del TLLC son variados

Tabla 1. Signos clínicos según patrón hemodinámico predominante del shock séptico en el niño

\begin{tabular}{|ll|}
\hline Shock frío & Shock caliente \\
\hline Llene capilar $>3^{\prime \prime}$ & Llenado capilar $\leq 2^{\prime \prime}$ \\
\hline Pulsos periféricos débiles & Pulsos periféricos amplios \\
\hline Extremidades frías & Extremidades calientes \\
\hline Presión de pulso angosta & Presión de pulso amplia \\
\hline
\end{tabular}

Tabla 2. Metas de reanimación clínica y de laboratorio para el paciente en shock séptico

\begin{tabular}{|ll|}
\hline Metas clínicas & Metas de laboratorio \\
\hline $\begin{array}{l}\text { Normalización de la frecuencia } \\
\text { cardíaca }\end{array}$ & Lactato $<2 \mathrm{mmol} / \mathrm{L}$ \\
\hline $\begin{array}{l}\text { Llenado capilar } \leq 2^{\prime \prime} \\
\text { Ausencia de diferencial de } \\
\text { pulsos centrales y periféricos }\end{array}$ & $\mathrm{SvcO}_{2} \geq 70 \%$ \\
\hline $\begin{array}{l}\text { Diuresis adecuada del déficit de base } \\
\text { Normalización del estado } \\
\text { mental }\end{array}$ \\
\hline $\begin{array}{l}\text { Normalización del shock index } \\
\text { Adecuada presión de perfusión }\end{array}$ \\
\hline
\end{tabular}

Shock index: frecuencia cardíaca (lat/min)/presión arterial sistólica $(\mathrm{mmHg}) ; \mathrm{SvcO}_{2}$, saturación venosa central de oxígeno; PCV, presión venosa central. 
e incluyen la resistencia arteriolar y venular, viscosidad sanguínea, eventual existencia de trombosis microvascular, policitemia, hiperleucocitosis, deshidratación y temperatura externa $^{39,42}$. Importantemente la presencia de fiebre no influye en el TLLC ${ }^{42}$.

Thompson et $\mathrm{al}^{43}$, señalaron que el reconocimiento de la prolongación del TLLC $(>2$ "), en conjunto con otros signos vitales puede mejorar el diagnóstico y tratamiento de niños con infecciones graves, con una sensibilidad comparable a diversos sistemas de triage pediátrico. Tibby et $\mathrm{al}^{44}$, describieron una correlación negativa entre el TLLC y volumen eyectivo y una correlación directa con los niveles de lactato en una población pediátrica de UCI. Asimismo en una comunicación reciente Raimer et al, demostraron una correlación inversa entre el TLLC y la saturación venosa de vena cava superior, es decir un TLLC $\leq 2$ " puede predecir una $\mathrm{SvcO}_{2} \geq 70 \%$. Por lo tanto, esta meta clínica puede ser relevante, principalmente en centros hospitalarios en donde la disponibilidad de acceso central es restringido ${ }^{45}$.

Se debe destacar que el TLLC es un indicador de la perfusión microvascular periférica, pero no del gasto cardíaco o flujo arterial. Esto se pudo corroborar en un diseño experimental pediátrico, donde la presión necesaria, generada mediante un manguito, para reducir en un 90\% el flujo arterial en la extremidad inferior, se relacionó con aumento de 0,89 segundos del TLLC, esto es muchísimo menos que la prolongación habitualmente observada en condiciones patológicas ${ }^{46}$.

El TLLC debe ser siempre evaluado en el contexto clínico y no en forma aislada, donde es escasamente útil.

\section{Acciones terapéuticas durante la primera hora de reanimación}

\section{Mantener o reestablecer vía aérea, oxigenación y ventilación}

El trabajo respiratorio y la compliance pulmonar cambia bruscamente durante el curso del SS. La decisión de intubación no se basa en resultados de laboratorio, sino en la evaluación clínica del paciente. Sobre el 40\% del gasto cardíaco está destinado al trabajo respi- ratorio, por lo tanto la intubación endotraqueal y conexión a ventilación mecánica debe ser prontamente efectuada.

El desarrollo de bradicardia durante la intubación endotraqueal se produce por estimulación vagal (hipoxia y/o estimulación laríngea). Se aconseja el uso de atropina en el SS, donde el anormal tono vasomotor existente puede transformar una bradicardia estable (respondedora a reoxigenación) en inestable (asociada con compromiso hemodinámico $)^{47}$. Otro fármaco que se puede utilizar es la ketamina, la que ofrece ventajas en el paciente con SS, pues se ha demostrado en modelos experimentales animales, que su administración inhibe las alteraciones hemodinámicas y la respuesta a citoquinas en el shock inducido por endotoxinas $^{48}$, como también mejorar la sobrevida al interferir con la cascada inflamatoria ${ }^{49}$. En relación al uso de etomidato, se debe tener precaución, pues es sabido que esta droga aumenta, transitoriamente, el riesgo de insuficiencia suprarrenal relativa ${ }^{50,51}$.

\section{Circulación}

La obtención de acceso vascular (vía venosa periférica) en la población pediátrica es más difícil que en adulto y aún más en situaciones de colapso hemodinámico, pero de seguir recomendaciones protocolizadas, ésta debiera obtenerse en la mayoría de los pacientes en los primeros cinco minutos de reanimación ${ }^{52}$. Una vez obtenido el acceso venoso se debe comenzar la resucitación con fluidos, y en caso que no se logren las metas mencionadas considerar el inicio de inótropos por esta vía.

\section{Resucitación con fluidos}

La reanimación con fluidos es una de las piedras angulares en el tratamiento del SS, siendo su fin restablecer la estabilidad hemodinámica y perfusión tisular ${ }^{24,53}$. Debido al disminuido volumen intravascular efectivo que presentan estos pacientes pueden ser indispensables grandes cantidades de fluidos de reanimación, pudiendo llegar en ocasiones hasta $200 \mathrm{ml} / \mathrm{kg}^{54,55}$. Estos elevados requerimientos de fluidos están asociados a un aumento en la sobrevida, sin aumentar la tasa de síndrome de distress respiratorio agudo o 
edema cerebral ${ }^{6,54}$. En la actualidad no existe consenso sobre cuál de los líquidos de reanimación (cristaloides vs coloides) podría ser mejor. Una reciente revisión sistemática no demostró evidencia suficiente sobre el uso preferencial de coloides o cristaloides, siendo los diversos tipos de soluciones empleadas igualmente efectivos si se utilizan precozmente y en la cantidad necesaria $(20 \mathrm{ml} / \mathrm{kg} \text { en } 10 \mathrm{~min} .)^{56}$. En relación al uso masivo de soluciones ricas en cloro, como suero fisiológico 0,9\% (154 $\mathrm{mEq} / \mathrm{L}$ ), su uso puede potenciar el desarrollo de acidosis metabólica. Asimismo, la elección de otras opciones de fluidos de reanimación puede modificar significativamente el estado ácido-base del paciente crítico ${ }^{57}$.

Oliveira et $\mathrm{al}^{58}$, demostraron que la mortalidad en niños con SS era mayor en quienes recibieron durante la primera hora menos de $40 \mathrm{ml} / \mathrm{kg}$ de líquido de reanimación y en los cuales el tratamiento no fue iniciado antes de 30 minutos después del diagnóstico de SS.

En un reciente y provocador estudio multicéntrico, Maitland et $\mathrm{al}^{59}$, evaluaron en más de 3.000 niños africanos que presentaban una infección grave con alteración de la perfusión, el uso de bolos de fluidos en la reanimación. Los autores concluyeron que la administración de bolos de fluidos aumentó la mortalidad. Estos resultados deben ser valorados con prudencia.

Son indicadores de una adecuada expansión con fluidos la normalización de los parámetros hemodinámicos. Empero, hay que considerar que la normalización de los signos vitales no es suficiente para determinar una adecuada respuesta al tratamiento. Rady et $\mathrm{al}^{60}$, demostraron en un grupo de 36 pacientes adultos con SS reanimados hasta la normalización de los signos vitales, que la mayoría de ellos (86\%) continuaban en un estado de hipoxia tisular global, evidenciado por una $\mathrm{SvcO}_{2}$ disminuida y niveles de lactato elevados.

Del mismo modo, Brierley y Peters ${ }^{61}$ señalaron en niños la existencia del "shock críptico", por la prevalencia de valores bajos de $\mathrm{SvcO}_{2}$, luego de una apropiada resucitación primaria con restauración de las metas habituales.

\section{Soporte hemodinámico}

El inicio de drogas vasoactivas debe reali- zarse si persisten signos de shock a pesar de una adecuada resucitación con fluidos. La droga de elección es la dopamina. No obstante, Ceneviva et $\mathrm{al}^{7}$, demostraron que los niños con shock refractario a fluidos, presentan distintos patrones hemodinámicos, incluyendo GC bajo/ Resistencia Vascular Sistémica (RVS) elevada (60\%), GC bajo/RVS baja (20\%) y GC elevado/RVS baja (20\%), por lo tanto, la elección de la droga a utilizar va a depender del patrón predominante. De la misma manera, Irazuzta et $\mathrm{al}^{62}$, insisten en estas diferencias y proponen usar vasopresores, inótropos y/o vasodilatadores en función de la condición hemodinámica del niño (SS frío o caliente). Ante la presencia de signos de shock frío la epinefrina es la elección y la norepinefrina si hay signos de shock caliente $^{4}$. En caso de hipodébito (evaluado por $\mathrm{SvcO}_{2}$ ) con normotensión, la droga de elección es la dobutamina ${ }^{63}$.

Se ha señalado que los diferentes patrones hemodinámicos del SS son causa dependiente, así infecciones asociadas a uso de CVC presentan predominantemente un patrón de SS caliente, no así la sepsis adquirida en la comunidad que presenta habitualmente índice cardíaco bajo (SS frío) ${ }^{61}$.

\section{Inicio de antibióticos}

El tratamiento antibiótico es una de las bases fundamentales del tratamiento de la sepsis $^{64}$, su cumplimiento guarda estrecha relación con el pronóstico del paciente ${ }^{4,65}$. En un trabajo clásico, Kumar et $\mathrm{al}^{4}$, demostraron en población adulta un aumento de la mortalidad en un $7 \%$ por cada hora transcurrida sin uso de antibiótico. Se debe comenzar siempre en la primera hora del reconocimiento del $\mathrm{SS}^{63}$, de forma intravenosa e inicialmente de carácter empírico y de amplio espectro. Antes del inicio de la antibioterapia se deben obtener los cultivos adecuados, pero ésto nunca debe retrasar el inicio del tratamiento ${ }^{63,66,67}$. Finalmente, la elección del antibiótico va a depender de factores del huésped como son los agentes etiológicos según edad, estado inmunológico del paciente, si la infección es adquirida en la comunidad o de carácter nosocomial, del antibiótico como son el grado de penetración a los tejidos, nivel de toxicidad y finalmente 
también de la susceptibilidad del patógeno a la terapia antibiótica ${ }^{34}$.

\section{Control del foco infeccioso}

Se debe evaluar la presencia de un foco de infección susceptible de erradicación. Su foco anatómico debe diagnosticarse dentro de las primeras seis horas ${ }^{63,68}$. Localizado el foco infeccioso se realizarán los procedimientos destinados a su erradicación.

\section{Corrección de alteraciones metabólicas}

La hipoglicemia e hipocalcemia deben ser corregidas $^{66}$.

Finalmente, se debe señalar que los errores más frecuentes en el tratamiento inicial del paciente séptico son ${ }^{69}$ :

1. Falla en establecer, de manera rápida, un acceso vascular seguro.

2. Resucitación inadecuada de fluidos (subóptima).

3. Retardo en la intubación endotraqueal.

4. Inadecuada terapia empírica antibiótica (pequeño espectro de cobertura).

5. Falla en el control del foco infeccioso.

6. Falla en reconocer sobrecarga de líquidos.

7. Falsa revaluación post resucitación.

\section{Conclusión}

El SS es un importante problema de salud pública que requiere de la implementación de medidas específicas dirigidas a tomar conciencia del problema, identificarlo precozmente, desarrollar pautas de actuación de acuerdo a los conocimientos actuales y facilitar su aplicación en la práctica clínica. La evolución de estos pacientes es tiempo dependiente, existiendo una "hora de oro" en la que la participación del equipo de salud del área de urgencias es trascendental.

El entrenamiento y la protocolización de la atención de estos pacientes ha demostrado una reducción importante en la mortalidad, sin embargo en muchas ocasiones el tratamiento no es tan precoz ni adecuado como se podría realizar, por lo tanto, junto con el desarrollo e instauración de nuevas terapias, el uso más efectivo y racional de las ya existentes debiera mejorar el pronóstico a futuro.

\section{Referencias}

1. Montalvan G: Shock séptico en pediatría. Revista Electrónica de Medicina Intensiva. Disponible en http:// remi.uninet.edu/2007/03/REMIA068i.html. Accedido 15 de abril de 2012.

2. Aneja R, Carcillo J: Differences between adult and pediatric septic shock. Minerva Anesttesiol 2011; 77: 1-7.

3. Carcillo J, Kuch B, Han Y, et al: Mortality and Functional Morbidity After Use of PALS/APLS by Community Physicians. Pediatrics 2009; 124; 500-8.

4. Kumar A, Roberts D, Wood KE, et al: Duration of hypotension before initiation of effective antimicrobial therapy is the critical determinant of survival in human septic shock. Crit Care Med 2006; 34: 1589-96.

5. Barie PS, Williams MD, McCollam JS, et al: Benefit/ risk profile of drotrecogin alfa (activated) in surgical patients with severe sepsis. Am J Surg 2004; 188: 21220.

6. Carcillo J, Fields A: Clinical practice parameters for hemodynamic support of pediatric and neonatal patients in septic shock. Crit Care Med 2002; 30: 1365-78.

7. Ceneviva G, Paschall JA, Maffei F, Carcillo JA: Hemodynamic support in fluid-refractory pediatric septic shock. Pediatrics 1998; 102: e19.

8. McKiernan CA, Lieberman SA: Circulatory shock in children: an overview. Pediatr Rev 2005; 26: 451-60.

9. Luce WA, Hoffman TM, Bauer JA: Bench-to-bedside review: Developmental influences on the mechanisms, treatment and outcomes of cardiovascular dysfunction in neonatal versus adult sepsis. Crit Care 2007; 11: 228.

10. Trzeciak $S$, Dellinger RP, Chansky ME, et al: Serum lactate as predictor of mortality in patients with infection. Intensive Care Med 2007; 33: 970-7.

11. Huckabee WE: Abnormal resting blood lactate, I. The significance of hyperlactatemia in hospitalized patients. Am J Med 1961; 30: 833.

12. Weil MH, Afifi AA: Experimental and clinical studies on lactate and pyruvate as indicators of the severity of acute circulatory failure (shock). Circulation 1970; 41: 989-1001.

13. Vitek V, Cowley RA: Blood lactate in the prognosis of various forms of shock. Ann Surg 1971; 173: 308-13.

14. Moomey CB Jr, Melton SM, Croce MA, et al: Prognostic value of blood lactate, base deficit, and oxygen-derived variables in an LD50 model of penetrating trauma. Crit 
Care Med 1999; 27: 154-61.

15. Peretz DI, Scott HM, Duff J, et al: The significance of lacticacidemia in the shock syndrome. Ann N Y Acad Sci 1965; 119: 1133-41.

16. Cowan BN, Burns HJG, Boyle P, et al: The relative prognostic value of lactate and hemodynamic measurement in early shock. Anaesthesia 1984; 39: 750-5.

17. Arnold RC, Shapiro NI, Jones AE, et al: Multicenter study of early lactate clearance as a determinant of survival in patients with presumed sepsis. Shock 2009; 32: 35-9.

18. Duke TD, Butt W, South M: Predictors of mortality and multiple organ failure in children with sepsis. Intensive Care Med 1997; 23: 684-92.

19. Koliski A, Cat I, Giraldi DJ, Cat ML: Blood lactate concentration as prognostic marker in critically ill children. J Pediatr (Rio J) 2005; 81: 287-92.

20. Hatherill M, Tibby SM, Evans R, Murdoch IA: Gastric tonometry in septic shock. Arch Dis Child 1998; 78 : 155-8.

21. Scott HF, Donoghue AJ, Gaieski DF, Marchese RF, Mistry $R D$ : The utility of early lactate testing in undifferentiated pediatric systemic inflammatory response syndrome. Acad Emerg Med 2012; 19: 1276-80.

22. Jat KR, Jhamb U, Gupta VK: Serum lactate levels as the predictor of outcome in pediatric septic shock. Indian J Crit Care Med 2011; 15: 102-7.

23. Montalván G, Morejón L, Varela O: Saturación venosa de oxígeno en el niño críticamente enfermo. Rev Cubana Pediatr 2009; 81, $\mathrm{n}^{\circ} 3$.

24. Rivers E, Nguyen B, Havstad S, et al: Early Goal- Directed Therapy Collaborative Group. Early goal directed therapy in the treatment of severe sepsis and septic shock. N Engl J Med 2001; 346: 1368-77.

25. Hernández $G, P e \tilde{a} a H$, Cornejo $R$, et al: Impact of emergency intubation on central venous oxygen saturation in critically ill patients: a multicenter observational study. Crit Care 2009; 13: R63.

26. Kissoon N, Orr R, Carcillo J: Updated American College of Critical Care Medicine -Pediatric Advanced Life Support Guidelines for Management of Pediatric and Neonatal Septic Shock. Relevance to the Emergency Care Clinician. Pediatr Emer Care 2010; 26: 867-9.

27. Donoso A, Arriagada D, Cruces P, Díaz F: Shock séptico en pediatría I. Enfoque actual en el diagnóstico y tratamiento. Rev Chil Pediatr 2013; 84: 484-98.

28. Cruz AT, Perry AM, Williams EA, Graf JM, Wuestner $E R$, Patel B: Implementation of goal-directed therapy for children with suspected sepsis in the emergency department. Pediatrics 2011; 127: e758-66.

29. Larsen G, Mecham N, Greenberg R: An emergency department septic shock protocol and care guideline for children initiated at triage. Pediatrics 2011; 127: e1585e92.

30. Fisher JD, Nelson DG, Beyersdorf H, Satkowiak LJ: Clinical spectrum of shock in the pediatric emergency department. Pediatr Emerg Care 2010; 26: 622-5.

31. Tibby SM, Hatherill M, Marsh MJ, Murdoch IA: Clinicians' abilities to estimate cardiac index in ventilated children and infants. Arch Dis Child 1997; 77: 516-8.

32. Carcillo J: Reducing the global burden of sepsis in infants and children: A clinical practice research agenda. Pediatr Crit Care Med 2005; 6: S157-64.

33. Nguyen H, Rivers E, Abrahamian F, et al: Severe sepsis and septic shock: review of the literature and emergency department management guidelines. Ann Emerg Med. 2006; 48: 28-54.

34. Arnal L, Stein F: Pediatric septic shock: Why has mortality decreased? -The utility of goal-directed therapy. Semin Pediatr Infect Dis 2003; 14: 165-72.

35. Han Y, Carcillo JA, Dragotta M, et al: Early reversal of shock is associated with improved outcome. Pediatrics 2003; 112: 793-9.

36. Booy R, Habibi P, Nadel J, et al: Meningococcal Research Group. Reduction in case fatality rate from meningococcal disease associated with health care delivery. Arch Dis Child 2001; 85: 386-90.

37. de Oliveira CF, de Oliveira DS, Gottschald AF, et al: ACCM/PALS haemodynamic support guidelines for paediatric septic shock: an outcomes comparison with and without monitoring central venous oxygen saturation. Intensive Care Med 2008; 34: 1065-75.

38. Carcillo JA: Capillary refill time is a very useful clinical sign in early recognition and treatment of very sick children. Pediatr Crit Care Med 2012; 13: 210-2.

39. Schriger $D L$, Baraff L: Defining normal capillary refill: variation with age, sex, and temperature. Ann Emerg Med 1988; 17: 932-5.

40. Strozik KS, Pieper CH, Roller J: Capillary refilling time in newborn babies: normal values. Arch Dis Child Fetal Neonatal Ed. 1997; 76: F193-6.

41. Crook J, Taylor RM: The agreement of fingertip and sternum capillary refill time in children. Arh Dis Child 2013; 98: 265-8.

42. Gorelick MH, Shaw KN, Murphy KO, Baker MD: Effect of fever on capillary refill time. Pediatr Emerg Care 1997; 13: 305-7.

43. Thompson M, Coad N, Harnden A, Mayon-White R, Perera $R$, Mant $D$ : How well do vital signs identify children with serious infections in paediatric emergency care? Arch Dis Child 2009; 94: 888-93. 
44. Tibby S.M, Hatherill M, Murdoch IA: Capillary refill and core-peripheral temperature gap as indicators of haemodynamic status in paediatric intensive care patients. Arch Dis Child 1999; 80: 163-6.

45. Raimer P, Yong H, Weber M, Annich G, Custer J: A normal capillary refill time of $\leq 2$ seconds is associated with superior vena cava oxygen saturations of $\geq 70 \%$. J Pediatr 2011; 158: 968-72.

46. Karpitskaya Y, Miller J, Otsuka NY: The influence of arterial flow on capillary refill in pediatric lower extremity. J Surg Orthop Adv 2008; 17: 74-6.

47. Jones P, Dauger S, Peters MJ: Bradycardia during critical care intubation: mechanisms, significance and atropine. Arch Dis Child 2012; 97: 139-44.

48. Taniguchi T, Shibata K, Yamamoto K: Ketamine inhibits endotoxin-induced shock in rats. Anesthesiology 2001; 95: 928-32.

49. Shaked G, Czeiger D, Dukhno O, et al: Ketamine improves survival and suppresses IL-6 and TNF alpha production in a model of Gram-negative bacterial sepsis in rats. Resuscitation. 2004; 62: 237-42.

50. Malerba G, Romano-Girard F, Cravoisy A, et al: Risk factors of relative adrenocortical deficiency in intensive care patients needing mechanical ventilation. Intensive Care Med 2005; 31: 388-92.

51. Den Brinker M, Hokken-Koelega AC, Hazelzet JA, de Jong FH, Hop WC, Joosten KF: One single dose of etomidate negatively influences adrenocortical performance for at least $24 \mathrm{~h}$ in children with meningococcal sepsis. Intensive Care Med 2008; 34: 163-8.

52. Kanter RK, Zimmerman JJ, Strauss RH, Stoeckel KA: Pediatric emergency intravenous access. Evaluation of a protocol. Am J Dis Child 1986; 140: 132-4.

53. Marik PE, Varon J: Sepsis: state of the art. Dis Mon 2001; 47: 465-532.

54. Carcillo JA, Davis AL, Zaritsky A: Role of early fluid resuscitation in pediatric septic shock. JAMA 1991; 266: 1242-5.

55. Parker MM, Shelhamer JH, Natanson C, Alling DW, Parrillo JE: Serial cardiovascular variables in survivors and nonsurvivors of human septic shock: Heart rate as an early predictor of prognosis. Crit Care Med 1987; 15 : 923-9.

56. Akech S, Ledermann H, Maitland K: Choice of fluids for resuscitation in children with severe infection and shock. BMJ 2010; 341: c4416.

57. Yunos NM, Kim IB, Bellomo R, et al: The biochemical effects of restricting chloride-rich fluids in intensive care. Crit Care Med 2011; 39: 2419-24.

58. Oliveira CF, Nogueira de Sá FR, Oliveira DS, et al:
Time-and fluid-sensitive resuscitation for hemodynamic support of children in septic shock: barriers to the implementation of the American College of Critical Care Medicine/Pediatric Advanced Life Support Guidelines in a pediatric intensive care unit in a developing world. Pediatr Emerg Care 2008; 24: 810-5.

59. Maitland K, Kiguli S, Opoka RO, et al: Mortality after fluid bolus in African children with severe infection. $\mathrm{N}$ Engl J Med 2011; 364: 2483-95.

60. Rady MY, Rivers EP, Nowak RM: Resuscitation of the critically ill in the ED: responses of blood pressure, heart rate, shock index, central venous oxygen saturation, and lactate. Am J Emerg Med 1996; 14: 218-25.

61. Brierley J, Peters MJ: Distinct hemodynamic patterns of septic shock at presentation to pediatric intensive care. Pediatrics 2008; 122: 752-9.

62. Irazuzta J, Sullivan KJ, García PC, Piva JP: Pharmacologic support of infants and children in septic shock. J Pediatr (Rio J) 2007; 83: S36-45.

63. Dellinger P, Levy M, Carlet J, Bion J, Paarker M, Jaeschke R: Surviving sepsis campaign: International guidelines for management of severe sepsis and septic shock: 2008. Crit Care Med 2008; 36: 296-327.

64. Bochud PY, Bonten M, Marchetti O, Calandra T: Antimicrobial therapy for patients with severe sepsis and septic shock: An evidence-based review. Crit Care Med 2004; 32: S495-512.

65. Gaieski DF, Mikkelsen ME, Band RA, et al: Impact of time to antibiotics on survival in patients with severe sepsis or septic shock in whom early goal-directed therapy was initiated in the emergency department. Crit Care Med 2010; 38: 1045-53.

66. Brierley J, Carcillo J, Choong K, et al: Clinical practice parameters for hemodynamic support of pediatric and neonatal septic shock: 2007 update from the American College of Critical Care Medicine. Crit Care Med 2009; 37: 666-88.

67. Leibovici L, Shraga I, Drucker M, Konigsberger H, Samra Z, Pitlik SD: The benefit of appropriate empirical antibiotic treatment in patients with bloodstream infection. J Intern Med 1998, 244: 379-86.

68. Moss RL, Musemeche CA, Kosloske AM: Necrotizing fascitis in children: prompt recognition and aggressive therapy improve survival. J Pediatr Surg 1996; 31: 1142-6.

69. Management of Severe Sepsis \& Septic Shock in Infants \& Children. Disponible en:http://www.uhs.nhs. uk/Media/SUHTInternet/Services/IntensiveCare/PICU/ SevereSepsisandSepticShockGuidelines.pdf. Accedido el 18 de febrero de 2012. 